\title{
Nonequilibrium Phase Transition in Scattered Cell Communities Coupled by Auto/Paracrine-Like Signalling
}

\author{
H. Berry
}

\begin{abstract}
Auto/paracrine cell-to-cell communications via diffusive messengers can be coupled to a positive feedback loop in which cell stimulation by a messenger results in the production of new messengers. This yields a potential mechanism for relay transmission of the emitted message. This paper investigates the influence of noise on this mutual coupling of the cells with their environment, using numerical simulations of a stochastic minimal model. The results demonstrate that the deterministic (mean-field) approximation of this stochastic process fails short of predicting its behaviour because of the presence of strong noise-induced fluctuations. Instead, the behaviour of the model can be explained by the occurrence of a nonequilibrium phase transition, which is found to be in the universality class of directed percolation. This provides a theoretical framework to understand signal transmission in these stochastic systems.
\end{abstract}

Keywords Signal transmission · autocrine relay · stochastic models · critical phenomena $\cdot$ directed percolation

\section{Introduction}

Complex behaviours in cell communities such as self-organization and emergent phenomena may result from coupling of the cells with an environment they dynamically modify. For instance, cells often respond to molecules in their environment via intracellular signalling pathways that eventually result in altered concentration of the very extracellular molecular species that triggered the pathway.

A well-known example is auto/paracrine signalling. In this paradigm, cells emit a peptidic factor (e.g. EGF) that diffuses in the extracellular space until it reaches a neighbouring cell (paracrine signaling) or the source cell that emitted it (autocrine signaling) (Wiley et al., 2003). Stimulation by the diffusive factor may in turn trigger

\footnotetext{
H. Berry

INRIA, Team Alchemy, Parc Club Orsay Université, 3, rue J. Rostand, 91893 Orsay Cedex France e-mail: hugues.berry@inria.fr
} 
intracellular signalling cascades (e.g. the MAPK pathway) that eventually lead to the release of new diffusive factor molecules in the environment (positive feedback loop) (Pribyl et al., 2003a; Freeman, 2000).

Strikingly, this process can be considered as a rather generic communication pattern, that is not restricted to members of the EGF family or cytokines, but is also encountered in cAMP-mediated communications between Dictyostelium discoideum cells; acylated homoserine lactone-mediated quorum sensing in Vibrio fischeri (James et al., 2000) and other bacteria (de Kievit \& Iglewski, 2000); or even airborne virus spreading - which has been implied in the dissemination of the foot-and-mouth disease (Gloster et al., 2005) or the influenza virus flu (Hammond et al., 1989) for instance.

Although dissimilar, these examples share common features. First, the messenger travels at random, through diffusional or nondirectional transport. Moreover, encounter with the messenger molecule alters cell functioning in such a way that new messengers are ultimately excreted. Finally, stimulation by the messenger molecules is usually followed by a refractory or lag period during which the cell does not respond to new encounters with messenger molecules.

Broadly speaking, this process can be thought of as implementing relay broadcasting: "if a message is received, relay it to one of your nearest neighbours". Hence, though the spatial range of a single messenger molecule may be limited (Shvartsman et al., 2001), the presence of the feedback loop may allow messages to be transmitted over long distances, very much like the spreading of epidemics. For instance, in a detailed deterministic continuous biophysical model, Pribyl et al. (2003a) showed that this kind of relay autocrine signalling may give rise to messenger travelling waves that spread over the entire cell population.

However, like many processes in cell biology, this mechanism comes with inherent noise or stochasticity at several levels. First, because of the diffusive nature of the messenger movements, the target cell of an emitted messenger is random, i.e. cannot be precisely specified. Secondly, because it relies on intrinsically stochastic biochemical reactions, the triggering of an intracellular signalling pathway upon cell-messenger molecule interaction is probabilistic. Finally, at every moment, a messenger molecule can be removed from the system, either by extracellular proteolysis, or by scavenging in the extracellular space.

In recent years, several papers have presented biophysical models for auto/ paracrine signalling. However, to the best of our knowledge, these studies consisted either in deterministic models of auto/paracrine relay transmission (Pribyl et al., 2003a,b) or in stochastic models for messenger-cell interactions, but without feedback relay loops (Batsilas et al., 2003; Shvartsman et al., 2001).

The goal of the present work is thus to study the influence of noise in auto/ paracrine-like relay broadcasting systems. In particular, it investigates the collective behaviour exhibited by the mutual coupling between cells and their environment, and how messages can be transmitted in stochastic conditions. In this framework, we focused on the basic mechanisms underlying these generic processes, and voluntarily restricted our model to the elementary ingredients identified above. The results demonstrate that classical deterministic modelling fails to predict the behaviour 
observed in stochastic simulations because of large fluctuations due to the intrinsic noise. Moreover, we show that the behaviour of this stochastic system is due to the occurrence of a nonequilibrium phase transition and present evidence that this nonequilibrium phase transition is in the universality class of directed percolation.

\section{The Model}

As already mentioned above, we are interested here in the most fundamental processes implied in auto/paracrine-like relay signalling. Hence, we study a "minimal" model that incorporates only the few basic ingredients enumerated above (messenger diffusion, feedback-positive loop for messenger relay, refractory phase). Furthermore, the model is intrinsically stochastic regarding cell stimulation by messenger molecules, as well as messenger molecule survival in the extracellular space. For the same reasons, we study a one-dimensional version of the model, i.e. messenger molecules and cells evolve in a one-dimensional lattice of linear size $L$ with periodic boundaries (i.e. a circle made of $L$ equidistant lattice sites).

\subsection{Messenger Molecules}

Messenger molecules undergo isotropic diffusion on the lattice via non-interacting nearest-neighbour random walks without excluded volume conditions (i.e. several messengers can occupy a same site at a given time). Every messenger can also be removed from the lattice with rate $\lambda$ per time step.

\subsection{Cells}

Besides messenger molecules, the lattice also contains $n$ immobile point cells that are regularly scattered over the lattice. Each cell occupies a single lattice site, so that, as $n<L$, the distance between two nearest-neighbour cells is several lattice sites. Each cell $i=1 \ldots n$ is associated with a dynamical variable $\phi_{\mathrm{i}}(t)$ that takes integer values between 0 and $K-1$ and represents the state of cell $i$ at simulation time step $t$. Upon stimulation by a messenger molecule, a cell enters a refractory phase which lasts $K-1$ time steps and during which the cell is not responsive to messenger molecules (see Fig. 1). The state $\phi_{\mathrm{i}}=0$ is the quiescent state. The transition from $\phi_{\mathrm{i}}=0$ to $\phi_{\mathrm{i}}=1$ represents the stimulation step and occurs with probability $\omega$ (per time step) if a messenger molecule is present on cell $i$ lattice site. Transition from the state $\phi_{\mathrm{i}}=j$ to $\phi_{\mathrm{i}}=j+1$ then occurs with probability 1 (at each time step) for $j=1 \ldots K-1$. After $K$ steps, the cell is back in the quiescent state i.e. goes from $\phi_{\mathrm{i}}=K-1$ to $\phi_{\mathrm{i}}=0$ with probability 1 . Finally, the transition from $\phi_{\mathrm{i}}=K_{\mathrm{p}}$ to $\phi_{\mathrm{i}}=K_{\mathrm{p}}+1\left(K_{\mathrm{p}} \in 1 \ldots K-1\right)$ is accompanied by the release of a new messenger on cell $i$ lattice site. 


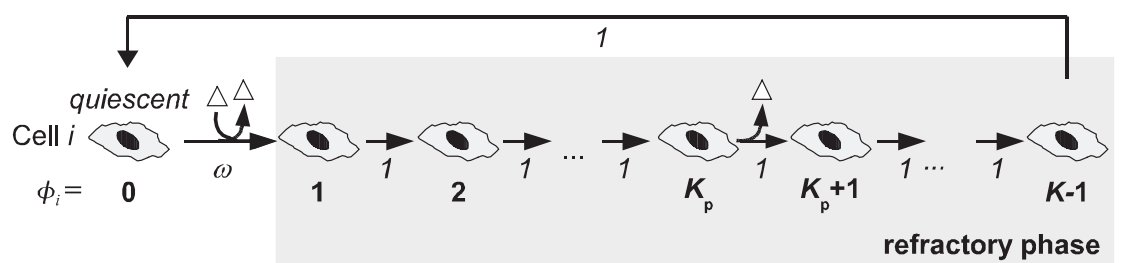

Fig. 1 Overview of the cell activation cycle. In the sequence, the cell state $\phi_{\mathrm{i}}$ is indicated by a bold number below the cell. Transition probabilities (at each time step) between two cell states are indicated by italicized numbers below corresponding arrows. Intervening messenger molecules are symbolized by white triangles. During the refractory phase of the cycle (light grey background), the cell is not sensible to messenger molecules

\subsection{Initialization}

The initial state of the system corresponds to "full lattice" initial conditions: one messenger molecule is initially positioned on each lattice site and the state of each cell $\phi_{\mathrm{i}}(0), \forall i=1 \ldots n$, is randomly chosen from a uniform distribution between 0 and $K-1$.

\subsection{Simulations}

Numerical simulations basically implement the above listed rules (Fig. 1, Sections 2.1. and 2.2). For clarity, we present below the translation of these rules in the point of view of the simulation algorithm.

After initialization, the system is updated as follows at each simulation step $t>0$ :

- The state $\phi_{\mathrm{i}}$ of each cell $i$ is first updated. If a messenger molecule is present on cell $i$ lattice site and if $i$ is quiescent $\left(\phi_{\mathrm{i}}(t-1)=0\right)$, its state is updated to $\phi_{\mathrm{i}}(t)=1$ with probability $\omega$. Note that, in the presence of $m$ messengers on the same cell site, the effective stimulation probability is $P\left(\phi_{\mathrm{i}}=0 \rightarrow \phi_{\mathrm{i}}=1\right)=$ $1-(1-\omega)^{m}$. Cells in the refractory phase $\left(\phi_{\mathrm{i}}(t-1)>0\right)$ are updated according to $\phi_{\mathrm{i}}(t)=\phi_{\mathrm{i}}(t-1)+1$ or $\phi_{\mathrm{i}}(t)=0$ if $\phi_{\mathrm{i}}(t-1)=K-1$. Finally, new messengers are created at each cell site for which the updated state variable $\phi_{\mathrm{i}}(t)=K_{\mathrm{p}}+1$.

- The messenger molecules are then updated according to two substeps. Each messenger is first independently and simultaneously moved to one of its two nearest-neighbour sites (chosen at random). Each molecule is then removed from the lattice with uniform probability $\lambda$.

\subsection{Parameter Values}

Unless otherwise stated, standard parameter values used in the present paper were: $L=500 \times 10^{3}$ sites, $n=50 \times 10^{3}$ cells, $K=20$ and $K_{\mathrm{p}}=10$. For each parameter 
set, the results presented are averages over 10 different realizations of the initial conditions and of the simulation run. The system behaviour was mainly studied through variations of the stimulation probability $\omega$ and the messenger removal probability $\lambda$.

\section{General Behaviour}

\subsection{Mean-Field Analysis}

To predict the behaviour of the model, a first approach neglects the inherently stochastic nature of the model and assumes that the fluctuations of the number of messenger molecules on the lattice are not significant. This so-called "meanfield" approach fundamentally approximates the model as a deterministic process, using differential equations that are actually similar to the mass-action laws used in classical (bio)chemical kinetics (see Berry (2003) for further details). Under these assumptions, the system is predicted to asymptotically reach a steady state (i.e. a stable fixed point). The messenger density $\rho(=m / L$ where $m$ is the total number of messenger molecules on the lattice) in the steady state is given by:

$$
\rho^{\mathrm{ss}}=\left\{\begin{array}{cl}
0 & \text { if } \omega<\omega_{c} \\
\frac{1}{K-1}\left(\frac{1}{\omega_{c}}-\frac{1}{\omega}\right) & \text { if } \omega \geq \omega_{c}
\end{array}\right.
$$

with

$$
\omega_{c}=\frac{\lambda L}{n}
$$

Hence, mean-field predictions state that, if $\omega<\omega_{c}$, messenger molecules are eventually cleared from the lattice (while every cell eventually becomes quiescent). In a way, this corresponds to the death of the system and will be referred to as the "absorbing phase". Conversely, if $\omega \geq \omega_{c}$, messenger molecules persist indefinitely on the lattice. This phase will be referred to as the "active phase".

When $\omega$ is close to $\omega_{c}$, a Taylor series expansion at first order of $\left(1 / \omega_{c}-1 / \omega\right)$ is $\left(\omega-\omega_{c}\right) / \omega_{c}^{2}$. Thus Eq. (1) can be rewritten as:

$$
\rho^{\mathrm{ss}} \propto \omega-\omega_{c}, \quad \text { for } \quad \omega \rightarrow \omega_{c} \text { in the active phase }
$$

Figure 2 presents simulation results illustrating the occurrence of the absorbing and active phases. In this figure, the $x$-axis is the 1-D lattice. A black dot is drawn at each lattice site containing a messenger molecule. The evolution of the system in time is plotted along the $y$-axis. When $\omega$ is small (Fig. 2A), messenger molecules persist for circa 100 time steps, before undergoing massive extinction. Eventually, after $\approx 600$ time steps, all the messengers have been removed from the lattice, and 

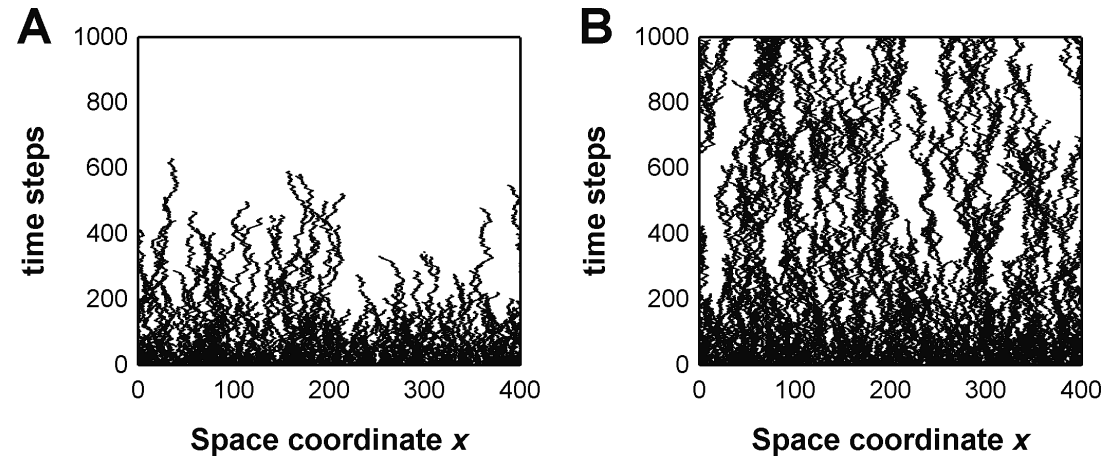

Fig. 2 Simulations of the model illustrating the occurrence of an absorbing (A) and an active (B) phase. Parameters were: $L=400$ sites, $n=40$ cells, $K=20, K p=10, \lambda=0.0150$ and (A) $\omega=0.100$ or $(\mathbf{B}) \omega=0.6235$

the system freezes into the absorbing (dead) phase. Conversely, for large $\omega$ values (Fig. 2B), the messenger molecules survive for the entire time span of the simulation (active phase). Albeit the system is constantly changing, visual inspection of the figure indicates that messenger density tends to a roughly constant value.

It must however be emphasized that the mean-field analysis above is valid only if the spatial fluctuations of messenger molecules are negligible. Yet simulations of the model such as those presented in Fig. 2 show that at every time step, the distribution of messenger molecules is rather inhomogeneous in space, with "white" zones (devoid of messengers) of various sizes and "black" regions where messenger density is high. This is a fundamental expression of the inherently stochastic nature of the model. It indicates that spatial fluctuations in the system are high, and could invalidate some of the predictions made by the deterministic mean-field analysis. To quantify and characterize the behaviour of the system with its natural stochasticity and fluctuations, intensive simulations of the model are needed. The following of the paper is devoted to these analyses.

\section{Simulation Results}

\subsection{Dynamics of the Messenger Density}

Figure 3 presents the time evolution for the density of messenger molecules $\rho$ in the active (Fig. 3A) or absorbing (Fig. 3B) phases. In the active phase, $\rho$ decreases according to three main regimes. After an initial slow decay regime (up to $\approx 20$ time steps), $\rho$ decreases abruptly for $\approx 10^{3}$ time steps. Finally, at long times, $\rho$ dynamics reaches a regime where it fluctuates around a constant average value. Fundamentally, this stationary fluctuating regime is the stochastic equivalent of the steady state predicted by the deterministic mean-field analysis (Eq. 1). The average value of the fluctuations at long times in the simulations will thus as well be noted $\rho^{\text {ss }}$. 

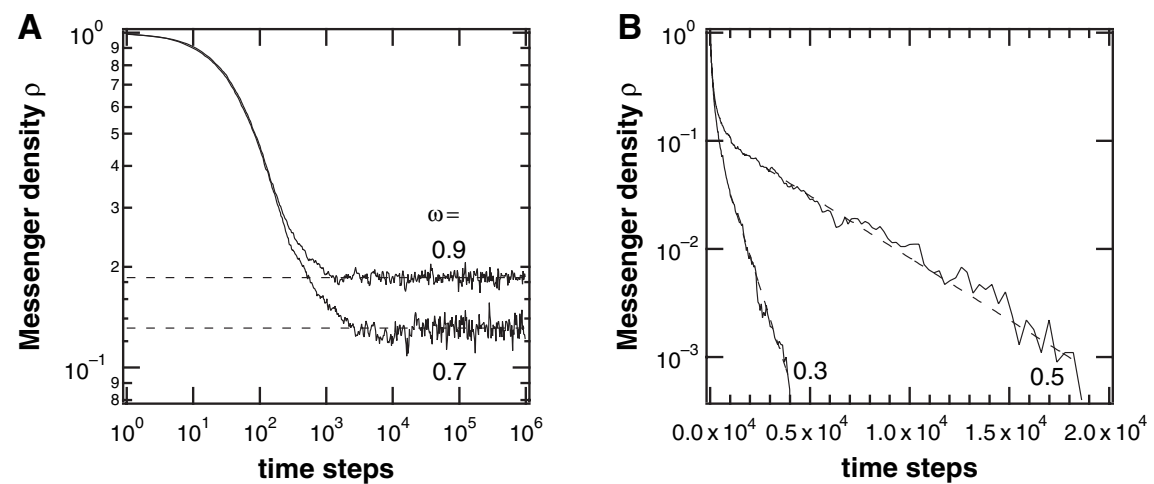

Fig. 3 Dynamics of the messenger density $\rho(t)$. Simulation results for "Full lattice" initial conditions in the active (A) or absorbing (B) phases. In (A) the dashed lines indicate the corresponding average value in the fluctuating stationary state $\rho^{\text {ss }}$. In $(\mathbf{B})$ dashed lines represent fits with exponentially decreasing functions. The values of $\omega$ are indicated beside the curves; $\lambda=0.0150$; $L=500 \times 10^{3}$ sites, $n=50 \times 10^{3}$ cells, $K=20$ and $K_{p}=10$. Results are from a single simulation run

Note however that, while both simulations and mean-field analysis indicate a stationary state in the active phase at long times, the predicted values are different. For instance, with the parameters of Fig. 3, Eq. (1) predicts $\rho^{\mathrm{ss}}=0.276$ (for $\omega=0.7$ ) and 0.292 (for $\omega=0.9$ ) while simulations yield 0.131 and 0.186 , respectively. This is a first evidence for failure of the mean-field analysis due to the high fluctuations in the model.

In the absorbing phase (Fig. 3B), the behaviour at long times is quite contrasted. Instead of reaching a stationary regime, messenger density vanishes very fast. Indeed, this curve is plotted in log-linear coordinates so that the observed linear behaviour indicates an exponentially fast decay.

Hence, in the active phase at long times, $\rho$ settles onto a fluctuating stationary regime, while in the absorbing phase, messengers vanish according to an exponential decay. The difference between these two behaviours can be used to identify the boundary between the two phases. Figure 4A shows the dynamics of $\rho$ for values of $\omega$ that are close to the transition between active and absorbing phases. For the smallest values of $\omega$ (the bottom curves in the figure), the curves bends down. Note that the curves are plotted here in log-log coordinates so that this downward curvature reflects the exponential decay characteristic of the absorbing phase. Conversely, the curves obtained for the largest values of $\omega$ (the upper curves in Fig. 4A) inflect upwards and tend towards the stationary state, which is a characteristic of the active phase.

At the boundary between the two phases, i.e. for the critical value $\omega_{c}$, the decay of $\rho$ at long times is thus expected to be linear in log-log coordinates (neither curving upwards nor downwards), which corresponds to the power-law:

$$
\rho(t) \propto t^{-\alpha}, \quad \text { for } \quad \omega=\omega_{c} \text {, and } t \rightarrow \infty \text {, }
$$



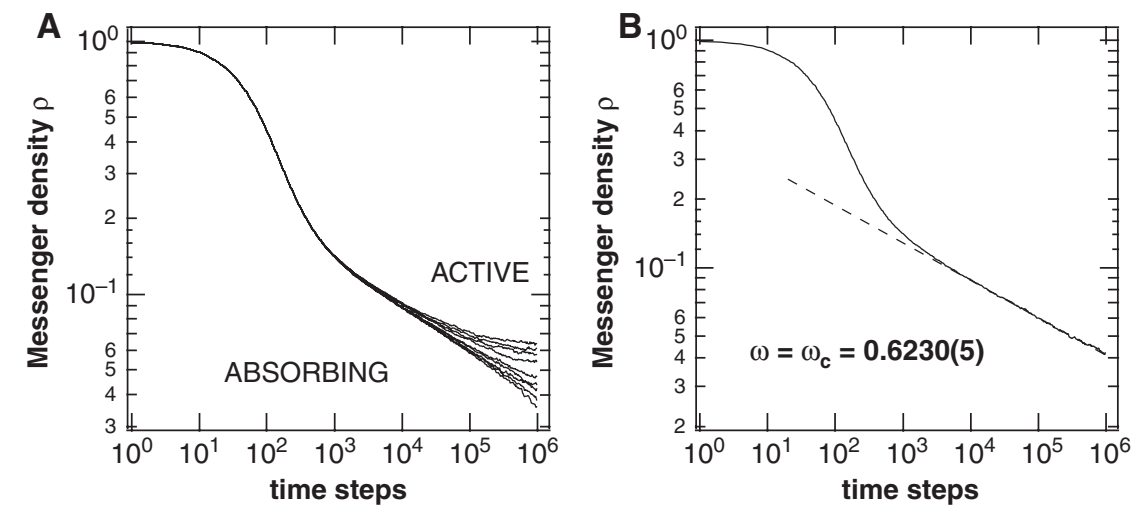

Fig. 4 Dynamics of the messenger density $\rho(t)$ close to the boundary between active and absorbing phases. (A) Each curve corresponds to a different value of $\omega$, i.e., from bottom to top, $\omega=0.6220,0.6225,0.6230,0.6235,0.6240,0.6260,0.6270,0.6280$ and 0.6290 . (B) Timevariation of $\rho$ when $\omega$ is set to its estimated critical value $\omega=\omega_{c}=0.6230(5)$. (The number between parentheses denotes the estimated incertitude on the last digit). The dashed curve indicates a power-law decay with exponent -0.165 . Parameters were $\lambda=0.0150$ and other parameters from the standard set (c.f. 2.5)

Hence, the value of $\omega$ that yields a power-law behaviour can be used as an estimate for $\omega_{c}$. Using this principle, Fig. 4B shows that an estimate of $\omega_{c}=0.6230$ is obtained. Furthermore the estimated value for the exponent of the corresponding power-law in this case is $\alpha \approx 0.165$. It must be noted that the prediction of the
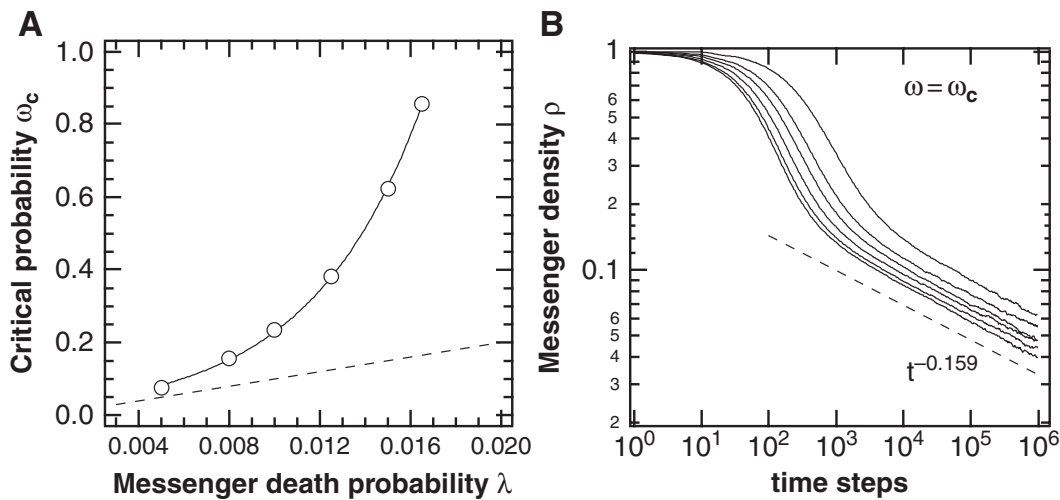

Fig. 5 Critical behaviour for various values of the messenger death probability $\lambda$. (A) Estimates of the critical stimulation probability $\omega_{\mathrm{c}}$ as a function of $\lambda$ (open circles). The dashed line indicates prediction from the mean-field analysis (Eq. 2). The full line is an exponential fit. (B) Dynamics of the messenger density $\rho(t)$ for various values of $\lambda$ at the corresponding critical value $\omega_{\mathrm{c}} . \lambda$ values are (from top to bottom): $0.0050 ; 0.0060 ; 0.0100 ; 0.0125 ; 0.0150$ and 0.0165 . The dashed line indicates a power-law decay with exponent 0.159 . All other parameters according to the standard values (Section 5) 
mean-field analysis fails here. In the case of Fig. 4, (Eq. 2) predicts $\omega_{c}=0.150$, which is less than one fourth of the value obtained in the simulations.

The critical behaviour for various values of the messenger death (removal) probability $\lambda$ is shown in Fig. 5. Simulations of the model show that the critical value $\omega_{c}$ increases exponentially fast with $\lambda$ (Fig. $5 \mathrm{~A}$, open circles). Here again, the prediction of the mean-field analysis (Fig. 5A, dashed line) clearly underestimates the actual value of the critical threshold. The dynamics of $\rho$ for various values of $\lambda$ at criticality (i.e. setting $\omega$ to its critical value $\omega_{c}$ at the corresponding value of $\lambda$ ) are plotted Fig. 5B. Albeit the final power-law regimes start at longer times when $\lambda$ decreases, all the curves obtained eventually reach a power-law regime with similar value of the exponent $\alpha$ (i.e. parallel straight lines in log-log coordinates). Averaging over these simulations, an improved estimate for the exponent $\alpha$ (Eq. 4) is obtained: $\alpha=0.159$ (7) (the number between parentheses indicates the estimated uncertainty on the last digit).

\subsection{A Nonequilibrium Phase Transition}

The dependence of the messenger density in the (fluctuating) stationary state $\rho^{\mathrm{ss}}$ on the stimulation probability $\omega$ is exemplified in Fig. 6 for $\lambda=0.0150$. The behaviour exhibited by $\rho^{\text {ss }}$ in the simulations is clearly typical of a (continuous or secondorder) phase transition between the active phase and the absorbing phase. It is for instance similar to the ferromagnetic phase transition observed in materials such as iron, where global magnetization increases continuously from zero as the temperature is lowered below the critical (Curie) temperature ${ }^{1}$.

However, a fundamental difference between the phase transition observed here and classical phase transitions lies in its nonequilibrium nature. Indeed, most of the classical phase transitions (including the ferromagnetic transition) are equilibrium phenomena, i.e. they obey the so-called "detailed balance" condition (Hinrichsen, 2006): the transition rate (or more precisely fluxes) from any state $i$ to any state $j$ is equal to that of the reverse transition rate, from $j$ to $i$. This condition implies the presence of real equilibrium states and is a fundamental property of these systems. In particular, it allows applying usual thermodynamic tools and concepts.

In the system studied here, the absorbing state induces a violation of the detailed balance condition: this state can be reached with some probability from the active state, but cannot be escaped. In turn, this prevents the application of several of the classical thermodynamic concepts. The general properties of these kinds of phase transitions, called nonequilibrium phase transitions, are thus still a mater of debate

\footnotetext{
${ }^{1}$ Note that the apparent discontinuity close to the critical value in Fig. 6 is a numerical artefact due to the so-called "critical slowing down". Indeed, as the critical threshold is approached, the fluctuating stationary regime starts at increasingly long simulation times. As this regime has to be reached to estimate the corresponding value of $\rho^{\text {ss }}$, the number of time steps in the simulations must be substantially increased as the critical threshold is approached. Hence the apparent discontinuity of Fig. 6 only reflects computational limitations hindering measurements with $\omega$ values closer to the threshold.
} 


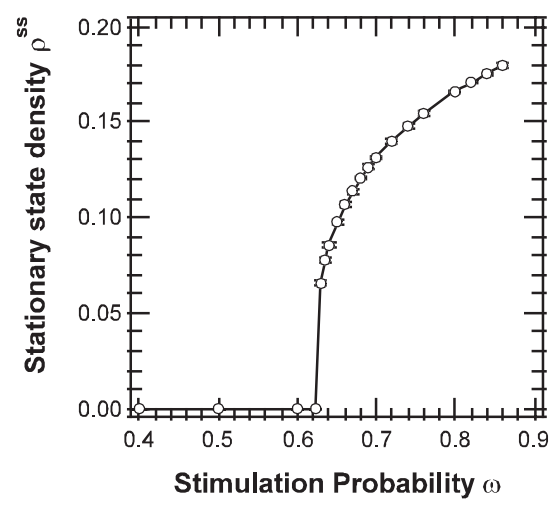

Fig. 6 Variation of the messenger molecule density in the (fluctuating) stationary state, $\rho^{\text {ss }}$, as a function of the stimulation probability $\omega$. Open circles show the results from the simulations of the models (the full line is a guide for the eyes). Parameters were $\lambda=0.0150$, and standard values for the others. Error bars show \pm 1 s.d.

in the physics community, but several points are well established (for reviews see Hinrichsen, 2006, Odor, 2004 or Hinrichsen, 2000a).

Like their equilibrium counterparts, nonequilibrium phase transitions exhibit generic power-law behaviours close to the critical threshold. Equation (4) is a first example of such a power-law, but we will encounter more of them in the following. Several quantities found in the power-laws depend on details of the simulation and the system considered: they are called "non-universal". For instance, the value of the critical threshold $\omega_{c}$ is known to be non-universal, and thus depends on the probability $\lambda$ for instance, as seen in Fig. 5A (just like the value of the percolation threshold depends on the type of percolation model considered, i.e. bond or link percolation). However, close to the critical threshold, several quantities (called universal quantities) are largely insensitive to microscopic details of the system (as in equilibrium phase transitions). For instance, the value of the exponent $\alpha$ (the critical exponent of power-law Eq. (4) does not vary when $\lambda$ changes (Fig. 5B).

One great success of the theory of equilibrium phase transitions is the explanation that transitions arising in different systems can share the same set of critical exponents. This phenomenon is called "universality". For example, the critical exponents at the liquid-gas critical point are independent of the chemical composition of the liquid. Furthermore, several models for transport in porous media (Sahimi, 1994) share the same critical exponent values as models of sol-gel transitions (Adam \& Lairez, 1996): they are in the same universality class (that of percolation, in this case). This means that the dominant processes implied in these systems are explainable by the same fundamental mechanisms.

Nonequilibrium phase transitions may as well be classified into several universality classes. Among them, the universality class of Directed Percolation (DP) has proven the most robust. Apparently very different systems, ranging from population dynamics (Lipowski \& Liposwka, 2000), epidemic spreading (Dammer \& 
Hinrichsen, 2003), forest fires (Albano, 1994), biological evolution (Ferreira \& Fontanari, 2002), $\mathrm{Ca}^{2+}$ signalling (Timofeeva \& Coombes, 2004; Bär et al., 2000) to morphology dynamics of growing surfaces (Hinrichsen, 2000a), belong to the DP universality class. This indicates that, beyond their diversity, all these systems rely fundamentally on the same ground processes. However, several other universality classes for nonequilibrium phase transitions have been uncovered, such as the parity conserving universality class (Zhong \& ben-Avraham, 1995), or the conserved threshold transfer process universality class (Lübeck \& Heger, 2003).

Hence, determining the universality class of a nonequilibrium phase transition is an important step towards the understanding of its fundamental mechanisms. Practically, this consists in estimating the value of the critical exponents of the system. This task is carried out in the following sections.

\subsection{Universality Class of the Phase Transition}

Besides Eq. (4), an important power-law for nonequilibrium phase transitions relates the average value of the density of messengers in the fluctuating stationary state, $\rho^{\mathrm{ss}}$, to the distance to the critical threshold, $\omega-\omega_{c}$ (Hinrichsen, 2006):

$$
\rho^{\mathrm{ss}} \propto\left(\omega-\omega_{c}\right)^{\beta}, \quad \text { for } \omega \rightarrow \omega_{c} \text { in the active phase }
$$

The corresponding critical exponent $\beta$ is a universal quantity. Actually Eq. (5) is identical to Eq. (3) with $\beta=1$, which means that the mean-field analysis predicts $\beta=1$. Figure 7 presents simulation results showing how $\rho^{\text {ss }}$ varies as a function of the distance to the threshold for various values of $\lambda$. These curves show roughly parallel straight lines at small distances, indicating a power-law behaviour with a critical exponent that does not depend on $\lambda$. Averaging over the 6 conditions of the figure, we obtain an estimate of $\beta=0.29(1)$. Here again, note that the value predicted by the mean-field analysis is more than threefold the measured one.

Another important critical exponent is the exponent related to the temporal correlations (see Section 5), $v_{\|}$. Theoretical arguments (Hinrichsen, 2006) indicate that plots of $\rho(t) \times t^{\alpha}$ as a function of $t \times\left(\omega-\omega_{c}\right)^{v \|}$ should yield a collapse of the curves shown Fig. 4A on two curves: every curves corresponding to active phase conditions should collapse to a single one, while those corresponding to absorbing phase conditions should collapse to another single one. Hence, finding the value of $v_{\|}$that yields the best collapse of the curves gives an estimate for this critical exponent.

Figure 8 shows the results obtained for $\lambda=0.0150$ (i.e. Fig. 4A). Using the estimated value of $\alpha=0.159$ (see above), the best collapse of the curves (at long times) was found for $v_{\|} \approx 1$.7. Similar treatments of the data for other values of $\lambda$ yielded comparable quantities (not shown). On average, the estimate for the critical exponent is $v_{\|}=1.66(9)$.

A third important critical exponent is the so-called dynamic exponent $z$. Roughly speaking, this exponent relates the behaviour of the system to the size of the spatial 


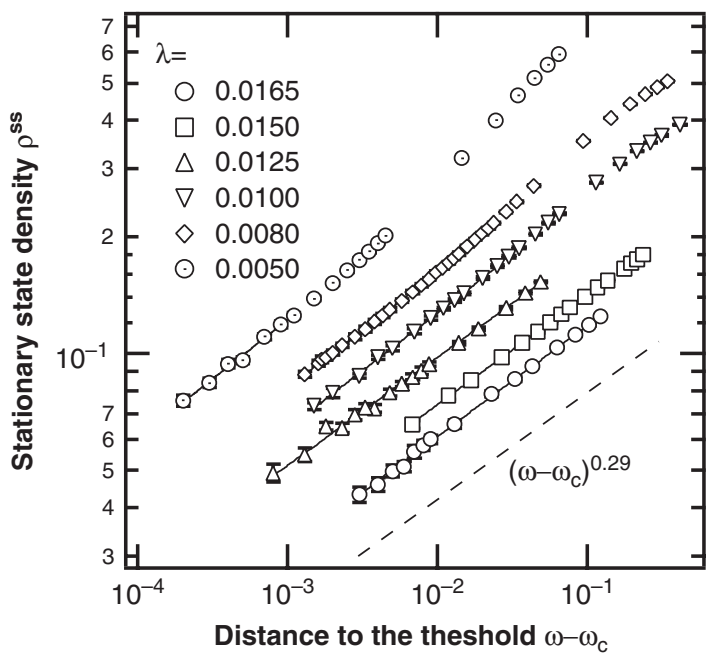

Fig. 7 Scaling of the messenger density in the fluctuating stationary states with the distance to the threshold for different values of the messenger death probability $\lambda$. The dashed line indicates a power law with exponent 0.29 . All other parameters are set to their standard values. Error bars show \pm 1 s.d.

domain (i.e. the number of sites $L$ in the lattice in our case) (Hinrichsen, 2006). It is important to realize that the various power-laws mentioned above are strictly valid for infinite spatial domains only, which is not accessible with computer simulations.

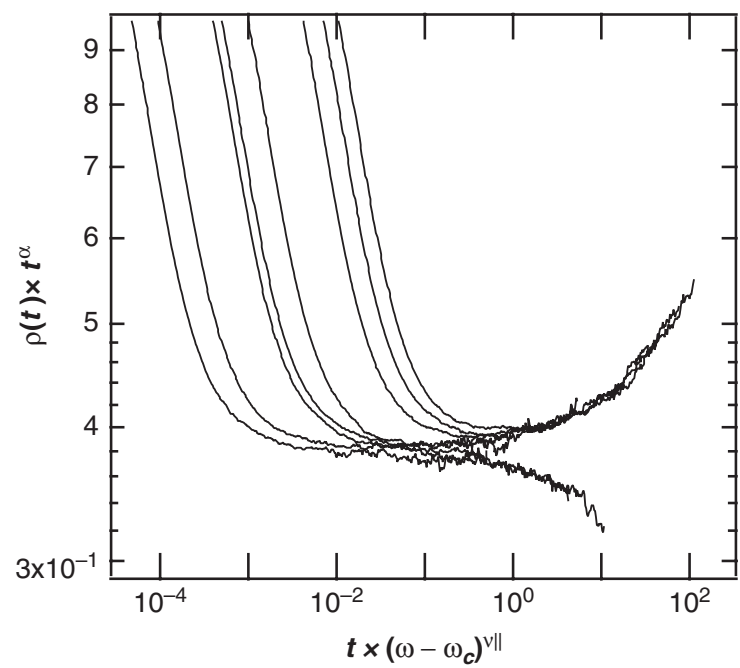

Fig. 8 Scaling test for the value of the critical exponent $v_{\|}$. The data of Fig. 4A are plotted here as $\rho(t) \times t^{\alpha}$ as a function of $t \times \omega-\left(\omega_{c}\right)^{v \|}$ with $\alpha=0.159$. The best collapse of the curves is obtained for $v_{\|}=1.70$. Parameters: $\lambda=0.0150$ and all other parameters according to their standard values 
The lattice size used in the simulations presented up to this point $\left(L=5 \times 10^{5}\right)$ was large enough so that the effects of this finite size could be neglected. In fact, for simulation durations of $10^{6}$ time steps, we have found that finite size effects are negligible as soon as $L \geq 5 \times 10^{3}$ sites.

Smaller $L$ values however evidence drastic effects. Figure 9A shows the dynamics of $\rho$ for small lattices $\left(L \leq 10^{3}\right)$ at criticality $\left(\omega=\omega_{c}\right)$ for $\lambda=0.0150$ and constant cell density (i.e. the number of cell is $n=0.10 \times L)$. In this case, the system has always a certain probability to jump into the absorbing phase (the corresponding curves bend downwards) even though the parameters correspond to the critical threshold (of course, this probability decreases when lattice size increases, and becomes very low for large but finite systems). Theoretical arguments (Hinrichsen, 2006) predict that in this case, plots of $\rho(t) \times t^{\alpha}$ as a function of $t / L^{z}$ should yield a collapse of the curves shown Fig. 9A on a single curve. As with $v_{\|}$above, this gives a method for estimating the dynamic exponent $z$. Fig. 9B shows the best collapse, obtained with $z=1.55$. The results of Fig. 9 can be replicated using other values for $\lambda$, and yield comparable estimates for $z$ (not shown). The average value obtained is $z=1.54(5)$.

Table 1 summarizes the values obtained for the four critical exponents estimated in this study. It also indicates an estimate for the critical exponent related to the spatial fluctuations, $v_{\perp}$. This exponent was not measured directly but deduced from the definition $z=v_{\|} / v_{\perp}$ (Hinrichsen, 2006). The table also indicates the corresponding values for the directed percolation (DP) universality class in one space dimension. Clearly, for each exponent, the obtained estimates are in good agreement with those of DP. This demonstrates that the nonequilibrium phase transition observed in the present model belongs to the directed percolation universality class.
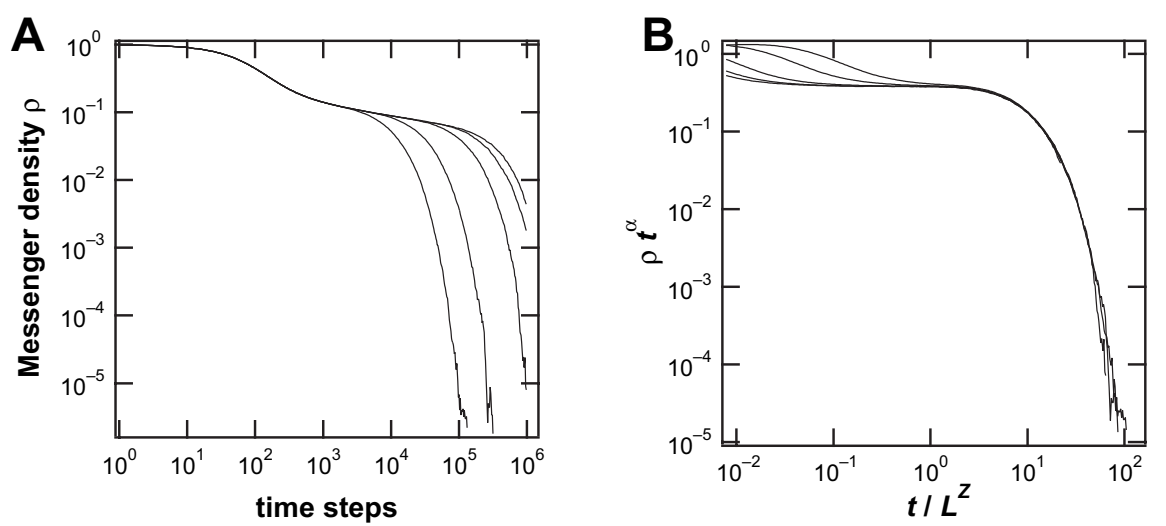

Fig. 9 Finite size scaling test at criticality $\left(\omega=\omega_{c}\right)$ and $\lambda=0.0150$. (A) dynamics of the messenger density $\rho(t)$ at criticality for different sizes of the lattice $L=100 ; 200 ; 500$; 800; and 1,000 (from bottom to top). Corresponding values of the number of cells $n=L / 10$ (constant cell density). (B) Scaling test for the value of the critical exponent $z$. The data of (A) are replotted here as $\rho(t) \times t^{\alpha}$ as a function of $t / L^{z}$ with $\alpha=0.159$. The best collapse of the curves is obtained for $z=1.55$. Parameters: $\lambda=0.0150$ and all other parameters according to their standard values 
Table 1 Comparison of the estimates obtained for the critical exponents studied in the present model and those of Directed Percolation (DP) in one space dimension (values are from Hinrichsen (2006)). Note that the value of $z$ for the present model is not a measure, but is deduced from $z=v_{\|} / v_{\perp}$. Numbers in parentheses indicate uncertainty in the last digit

\begin{tabular}{llllll}
\hline Model & $\alpha$ & $\beta$ & $v_{\|}$ & $z$ & $v_{\perp}$ \\
\hline Present & $0.159(7)$ & $0.29(1)$ & $1.66(9)$ & $1.54(5)$ & 1.08 \\
DP & $0.159464(6)$ & $0.276486(8)$ & $1.733847(6)$ & $1.580745(10)$ & $1.096854(4)$ \\
\hline
\end{tabular}

\section{Message Broadcasting}

The characterization of the phase transition allows a better understanding and prediction of several aspects of the model, such as the characteristics of message broadcasting. Let us imagine that the lattice is in the absorbing (dead) phase, i.e. devoid of any messenger molecule, with all cells quiescent. At time $t_{0}$, a single messenger molecule is produced by a cell at a random position on the lattice (see corresponding simulations in Fig. 10). Will this embryo of a message persist over time? Will it finally be transmitted to every cell in the lattice? In physicists' terms, this raises the question of the correlation length in the system. It turns out that, unlike their equilibrium counterparts, nonequilibrium phase transitions possess two independent correlation lengths: a spatial correlation length $\xi_{\perp}$, and a temporal correlation length $\xi_{\|}$(Hinrichsen, 2000a, 2006). These lengths are directly related to the spreading of the message seed.
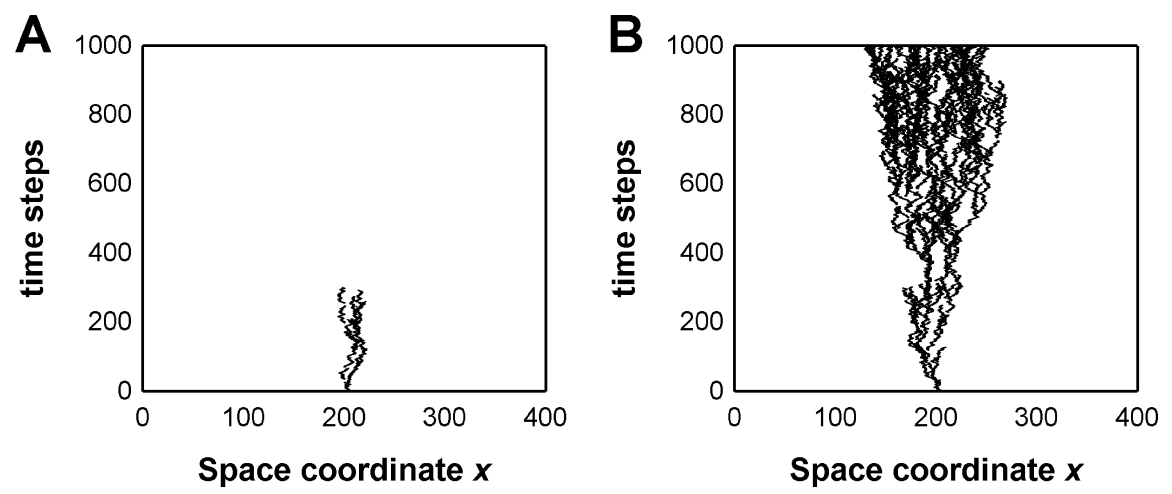

Fig. 10 Simulations of the model illustrating the spreading of a unique initial messenger "seed" in the absorbing phase $(\mathbf{A}) \omega=0.50$ or in the active phase $(\mathbf{B}) \omega=0.95$. Parameters were: $L=400$ sites, $n=40$ cells, $K=20, K p=10, \lambda=0.0150$. Each cells were initiated in the quiescent state $\left(\phi_{\mathrm{i}}(0)=0 ; \forall i=1 \ldots n\right)$

In the absorbing phase, the message survives for a certain amount of time and tends to slowly expand in space (Fig. 10A). The theory of nonequilibrium phase transition tells us that the average survival time is given by (or at least proportional to) $\xi_{\|} \propto\left|\omega-\omega_{c}\right|^{-v \|}$ and the maximal expansion of the message in space by $\xi_{\perp} \propto\left|\omega-\omega_{c}\right|^{-v \perp}$ (Hinrichsen, 2000a). Similarly, in the active phase, message 
spreads as a cone (Fig. 10B) whose opening angle is given by $\xi_{\perp} / \xi_{\|}$. Using these theoretical concepts, it becomes easy to understand that, as the critical threshold is approached from below in the simulations, the message spreading from the initial seed survives for increasing times and reaches a growing fraction of the cells. At the critical threshold, the effect of a single seed is even eventually transmitted (relayed) all along the lattice, whatever the physical distance to the initial seed site (both $\xi_{\|}$and $\xi_{\perp} \rightarrow \infty$ ). As $\omega$ increases further (above the threshold) and enters the active phase, the opening angle of the spread cone grows as $\left|\omega-\omega_{c}\right|^{\nu \|-v \perp}$. Thus, as $v_{\|}-v_{\perp}=0.637>0$ in DP, the time needed for the message to invade the whole lattice decreases with increasing $\omega$. Hence identifying the phase transition allows predicting both qualitatively and quantitatively the behaviours shown by the simulations.

\section{Discussion}

The idea that the stochasticity inherent to cellular processes has a strong influence on elementary behaviours such as toggle switch properties (Tian \& Burrage, 2006; Vilar et al., 2003), clock oscillations (Suel et al., 2006) or signal transduction (Bhalla, 2004), has recently emerged as a new paradigm in cell biology (for a review, see Rao et al., 2002). Furthermore, the role played by noise and stochasticity at the cell or organism level is beginning to be realized (for recent reviews see Samoilov et al., 2006 or Raser \& O’Shea, 2005).

The results of the present work add auto/paracrine-like relay signalling to the list of basic cell processes that may be highly influenced by noise-induced fluctuations. Our main objective was to study the influence of noise in a model for stochastic communications where cells respond to a diffusive environment that they themselves have produced through a positive feedback loop. Using numerical simulations, we showed that the deterministic (mean field) approximation of this process fails short of predicting the behaviour of the system. Instead, the latter could be understood as a nonequilibrium phase transition that was evidenced to be in the universality class of directed percolation.

That the model belongs to the directed percolation universality class is actually not totally unexpected. Indeed, DP has been conjectured (Grassberger, 1982) to be the universality class of models exhibiting a phase transition from a fluctuating active phase to a unique absorbing phase; whose dynamic rules involve only short-range processes; and that have no unconventional attributes such as additional symmetries (the actual conditions are in fact a bit more restrictive, see Hinrichsen, 2000a). The present model verifies these conditions thus supporting this conjecture.

Modelling studies of auto/paracrine relay transmission have rarely been addressed so far. In two studies, Pribyl and coworkers (Pribyl et al., 2003a,b) proposed a mechanistic model that presents similarities with the model studied here. Their model takes messenger diffusion, reversible binding to cell surface receptors and a positive feedback from ligand binding to new ligand release. The main differences 
with our model are that the feedback mechanism in their model is slightly more detailed (from a biochemical perspective) and most notably, their model is purely deterministic. Hence, from a stochastic point of view, this model could be considered as a mean-field limit. These authors show that their model supports travelling wave solutions that spread throughout the cell layer. In these waves, the front connects a steady state with nonzero messenger density to a steady state devoid of messengers. These behaviours are at first sight reminiscent of the spreading waves encountered in directed percolation, for instance for "seed" initial conditions (see Section 5), and of the active and adsorbing stationary states. Whether this model is related to directed percolation is however not trivial and would necessitate further works. For instance, the spreading waves in DP are not travelling waves (i.e. the shape of the wave front changes during its propagation). Starting from the discrete version of their model (Pribyl et al., 2003b), biologically realistic stochastic processes could be introduced at several points. Possible phase transitions and universality class of the resulting model could then be assessed through the study of the time evolution of the probability $P(t)$ that a spreading waves survives up to time $t$, that scales in the subcritical regime as $P(t) \sim t^{-\delta}$ with $\delta=0.1595$ or 0.451 for DP in $d=1$ or 2 dimensions, for instance.

Biological interpretation of the present work must be handled with great care. Indeed, the aim of the present work was to study the basic mechanisms at play in such kinds of diffusive communications, so that we voluntarily restricted the model to a set of a few elementary ingredients. Of course, this limits the biological realism of the model, and raises the question of the persistence of the observed behaviours in more detailed and biologically plausible models. While this question can only be answered by further modelling and simulation works, we give here some remarks related to this point.

First of all, the universality class of a phase transition is, by definition, not changed by details of the model. And directed percolation, in particular, has proven a very robust universality class to this respect. Hence, it seems unlikely that minor modifications in the model (such as changing the lattice geometry or using off-lattice conditions, using stochastic refractory periods for the cells...) would modify drastically the observed behaviour. For instance, preliminary investigations showed that changing the values of the cycle parameters $\left(K\right.$ and $K_{\mathrm{p}}$ ) modifies the value of the critical threshold but not the transition itself. Actually, shorter cycle lengths $K$ (not shown) decrease the duration of the initial phase i.e. the non power-law regime of $\rho$ dynamics (the first $10^{3}$ time steps in Fig. 4B).

An important parameter is the Euclidean dimension of the lattice. We choose here to study a one-dimensional model because 1-D models are much cheaper in computation time, thus allowing intensive simulations. However, biological realism would rather impose two-(or three-)dimensional spaces. Actually a twodimensional version of the model presented here has previously been studied in Berry, (2003). However, because simulations in this previous paper were not refined enough, the universality class could not be determined unambiguously. Hence, future work will focus on intensive simulations of the model in two and three space dimensions. 
Furthermore, another significant assumption in the present work is the regular spacing of the cells on the lattice. However, preliminary simulations (not shown) indicate that random cell positioning does not modify the occurrence of the nonequilibrium phase transition, but has the deleterious effect that the density of messenger molecules decays as a power-law in the absorbing phase (and not exponentially, as observed for regular spacing) ${ }^{2}$. This invalidates the estimation of the critical threshold based on the criterion of regime change between subcritical and critical conditions, that was used in Fig. 4, for instance. While more computationally expensive, other methods based on finite-size effects for instance (Ortega et al., 1998; Binder \& Heermann, 1997) can however be applied in this case and will be used in future studies of the model with random cell positioning.

A surprising point concerning directed percolation is that in spite of its amazing robustness in models/simulations, this critical behaviour has still not been evidenced in experiments (see Hinrichsen, 2000b). Hence, the possibility that autocrine relays may provide experimental evidence of DP should be considered seriously. Possible experimental setups could for instance consist in cell and tissue culture assays, where a confluent (or subconfluent) layer of autocrine cells is covered by a liquid medium, in which the soluble messenger is secreted and diffuses. Alternatively, such a situation is naturally found in Drosophilia eggs (see Pribyl et al., 2003b). The evolution of messenger concentration in the liquid or of related reporters could be monitored in situ, for instance using fluorescence. Such in situ recordings of spatio-temporal evolutions are already accessible to cell biologists (see e.g. Nikolic et al., 2006). Now, messenger death probability ( $\lambda$ in the present model) could be modulated through the addition of various levels of proteinases (degrading the messenger) in the liquid medium. Likewise, the stimulation probability $\omega$ could be modulated through the addition of various levels of antagonists of the messenger receptor, or intracellular inhibitors of the signalling pathway supporting the positive feedback. Finally, the A431 carcinoma cell line could be a good candidate for these studies (Dent et al., 1999).

\section{References}

Adam M., \& Lairez, D., 1996, Sol-gel transition. In Physical Properties of Polymeric Gels (J.P. Cohen Addad, ed.), John Wiley \&. Sons, UK, pp. 87-142.

Albano, E.V., 1994, Critical behaviour of a forest fire model with immune trees, J. Phys. A 277: L881-L886.

Bär, M., Falcke, M., Levine, H., \& Tsimring, L.S., 2000, Discrete stochastic modeling of calcium channel dynamics, Phys. Rev. Lett. 84:5664-5667.

Batsilas, L., Berezhkovskii, A.M., \& Shvartsman, S.Y., 2003, Stochastic model of autocrine and paracrine signals in cell culture assays, Biophys. J. 85:3659-3665.

Berry, H., 2003, Nonequilibrium phase transition in a self-activated biological network, Phys. Rev. E 67:031907.

\footnotetext{
2 Note that this phenomenon has already been encountered in the literature; see Szabo et al. (2002) for instance.
} 
Bhalla, U.S., 2004, Signaling in small subcellular volumes. I. Stochastic and diffusion effects on individual pathways, Biophys. J. 87:733-744.

Binder, K., \& Heermann, D.W., 1997, Monte Carlo Simulation in Statistical Physics, Springer, Berlin, Germany.

Dammer, S.M., \& Hinrichsen, H., 2003, Epidemic spreading with immunization and mutations, Phys. Rev. E 68:016114.

De Kievit, T.R., \& Iglewski, B.H., 2000, Bacterial quorum sensing in pathogenic relationships, Infect. Imun. 68:4839-4849.

Dent, P., Reardon, D.B., Park, J.S., Bowers, G., Logsdon, C., Valerie, K., Schmidt-Ullrich, R., 1999, Radiation-induced release of transforming growth factor alpha activates the epidermal growth factor receptor and mitogen-activated protein kinase pathway in carcinoma cells, leading to increased proliferation and protection from radiation-induced cell death, Mol. Biol. Cell 10:2493-2506.

Ferreira, C.P., \& Fontanari, J.F., 2002, Nonequilibrium phase transition in a model for the origin of life, Phys. Rev. E 65:021902.

Freeman, M., 2000, Feedback control of intercellular signalling in development, Nature 408:313-319.

Gloster, J., Freshwater, A., Sellers, R.F., \& Alexandersen, S., 2005, Re-assessing the likelihood of airborne spread of foot-and-mouth disease at the start of the 1967-1968 UK foot-and-mouth disease epidemic, Epidemiol. Infect. 133:767-783.

Grassberger, P., 1982, On phase transitions in Schlögl's second model, Z. Phys. B 47:365-374.

Hammond, G.W., Raddatz, R.L., \& Gelskey, D.E., 1989, Impact of atmospheric dispersion and transport of viral aerosols on the epidemiology of influenza, Rev. Infect. Dis. 11:494-497.

Hinrichsen, H., 2006, Non-equilibrium phase transitions, Physica A 369:1-28.

Hinrichsen, H., 2000a, Nonequilibrium critical phenomena and phase transitions into absorbing states, Adv. Phys. 49:815-958.

Hinrichsen, H., 2000b, On possible experimental realizations of directed percolation, Braz. J. Phys. 30:69-82.

James, S., Nilsson, P., James, G., Kjelleberg, S., \& Fagerström, T., 2000, Luminescence control in the marine bacterium Vibrio fischeri: An analysis of the dynamics of lux regulation, J. Mol. Biol. 296:1127-1137.

Lipowski, A., \& Liposwka, D., 2000, Nonequilibrium phase transition in a lattice prey-predator system, Physica A 276:456-464.

Lübeck, S., \& Heger, P.C., 2003, Universal finite-size scaling behavior and universal dynamical scaling behavior of absorbing phase transitions with a conserved field, Phys. Rev. E 68:056102.

Nikolic, D., Boettiger, A., Bar-Sagi, D., Carbeck, J., \& Shvartsman, S., 2006, Role of boundary conditions in an experimental model of epithelial wound healing, Am. J. Physiol. Cell Physiol. 291:C68-C75.

Odor, G., 2004, Universality classes in nonequilibrium lattice systems, Rev. Mod. Phys. 76:663-724.

Ortega, N.R.S., Pinheiro, F.S., Tomé, T., \& Drugowich de Felicio, J.R., 1998, Critical behavior of a probabilistic cellular automaton describing a biological system, Physica A 255:189-200.

Pribyl, M., Muratov, C.B., \& Shvartsman, S.Y., 2003a, Long-range signal transmission in autocrine relays, Biophys. J. 84:883-896.

Pribyl, M., Muratov, C.B., \& Shvartsman, S.Y., 2003b, Discrete models of autocrine cell communication in epithelial layers, Biophys. J. 84:3624-3635.

Rao, C.V., Wolf, D.M., \& Arkin, A.P., 2002, Control, exploitation and tolerance of intracellular noise, Nature 420:231-237.

Raser, J.M., \& O'Shea, E.K., 2005, Noise in gene expression: origins, consequences, and control, Science 309:2010-2013.

Sahimi, M., 1994, Applications of Percolation Theory, Taylor \& Francis, UK.

Samoilov, M.S., Price, G., \& Arkin, A.P., 2006, From fluctuations to phenotypes: the physiology of noise, Sci. STKE 366:re17. 
Shvartsman, S.Y., Wiley, H.S., Deen, W.M., \& Lauffenburger, D.A., 2001, Spatial range of autocrine signalling: modelling and computational analysis, Biophys. J. 81:1854-1867.

Suel, G.M., Garcia-Ojalvo J., Liberman, L.M., Elowitz, M.B., Tian, T., \& Burrage, K., 2006, An excitable gene regulatory circuit induces transient cellular differentiation, Nature 440:545-550.

Szabo, G., Gergely, H., \& Oborny, B., 2002, Generalized contact process on random environments, Phys. Rev. E 65:066111.

Tian, T., \& Burrage, K., 2006, Stochastic models for regulatory networks of the genetic toggle switch, Proc. Natl. Acad. Sci. USA 103:8372-8377.

Timofeeva, Y., \& Coombes, S., 2004, Directed percolation in a two-dimensional stochastic firediffuse-fire model, Phys. Rev. E 70:062901.

Vilar, J.M., Guet, C.C., \& Leibler, S., 2003, Modeling network dynamics: the lac operon, a case study, J. Cell Biol. 161:471-476.

Wiley, H.S., Shvartsman, S.Y., \& Lauffenburger, D., 2003, Computational modeling of the EGFreceptor systems: a paradigm for systems biology, Trends Cell Biol. 13:43-50.

Zhong, D., \& ben-Avraham, D., 1995, University class of two-offspring branching-annihilating random walks, Phys. Lett. A 209:333-337. 